\title{
The Analysis of Thin Wires Using Higher-Order Elements and Basis Functions
}

N. J. Champagne, D. R. Wilton, J. W. Rockway

February 14, 2006

IEEE AP-S International Symposium Albuquerque, NM, United States July 9, 2006 through July 14, 2006 
This document was prepared as an account of work sponsored by an agency of the United States Government. Neither the United States Government nor the University of California nor any of their employees, makes any warranty, express or implied, or assumes any legal liability or responsibility for the accuracy, completeness, or usefulness of any information, apparatus, product, or process disclosed, or represents that its use would not infringe privately owned rights. Reference herein to any specific commercial product, process, or service by trade name, trademark, manufacturer, or otherwise, does not necessarily constitute or imply its endorsement, recommendation, or favoring by the United States Government or the University of California. The views and opinions of authors expressed herein do not necessarily state or reflect those of the United States Government or the University of California, and shall not be used for advertising or product endorsement purposes. 


\title{
The Analysis of Thin Wires Using Higher-Order Elements and Basis Functions
}

\author{
Nathan J. Champagne ${ }^{(1)}$, Donald R. Wilton ${ }^{(2)}$ and John W. Rockway ${ }^{(3)}$ \\ (1) Louisiana Tech University, Ruston, LA 71272, USA \\ (2) University of Houston, Houston, TX 77204-4005, USA \\ (3) Lawrence Livermore National Laboratory, Livermore, CA 94550, USA ${ }^{\dagger}$
}

\section{Introduction}

Thin wire analysis was applied to curved wire segments in [1], but a special procedure was needed to evaluate the self and near-self terms. The procedure involved associating the singular behavior with a straight segment tangent to the curved source segment, permitting use of algorithms for straight wires. Recently, a procedure that avoids the singularity extraction for straight wires was presented in [2-4]. In this paper, the approach in [4] is applied to curved (or higher-order) wires using a procedure similar to that used in [1] for singularity extraction. Here, the straight tangent segment is used to determine the quadrature rules to be used on the curved segment. The result is a formulation that allows for a general mixture of higher-order basis functions [5] and higher-order wire segments.

\section{Method of Moments Analysis}

The currents on a wire structure are determined using the electric field integral equation (EFIE). The mixed potential formulation is used to determine the scattered electric field. Following the formulation in [1], the potentials are written in terms of partial potentials as

$$
\mathbf{A}(\mathbf{r})=\mu \sum_{n=1}^{N} I_{n} \int_{\sigma} \boldsymbol{\Lambda}_{n}\left(\mathbf{r}^{\prime}\right) K\left(\mathbf{r}, \mathbf{r}^{\prime}\right) d \sigma^{\prime}
$$

and

$$
\Phi(\mathbf{r})=\frac{-1}{j \omega \varepsilon} \sum_{n=1}^{N} I_{n} \int_{\sigma} \nabla_{S}^{\prime} \cdot \boldsymbol{\Lambda}_{n}\left(\mathbf{r}^{\prime}\right) K\left(\mathbf{r}, \mathbf{r}^{\prime}\right) d \sigma^{\prime},
$$

where the kernel is given by

$$
K\left(\mathbf{r}, \mathbf{r}^{\prime}\right)=\frac{1}{2 \pi} \int_{-\pi}^{\pi} \frac{e^{-j k R}}{4 \pi R} d \phi^{\prime} .
$$

Here, $d \sigma^{\prime}$ is the differential arc length along the wire axis $\sigma$ and $d \phi^{\prime}$ is an angle differential measured about the wire circumference.

There are no issues associated with using (1)-(3) when the source and observation points are well separated. However, when the observation point approaches the

\footnotetext{
${ }^{\dagger}$ This work was performed under the auspices of the U.S. Department of Energy by the University of California Lawrence Livermore National Laboratory under contract No. W-7405-ENG-48.
} 
source point, the wire kernel becomes singular. With the recent transformation presented in [2-4], singularity extraction is no longer needed since the inner integral's integrand is smoothed by variable transformations, whereas the logarithmically singular outer integral may be integrated numerically using the scheme of [4]. This scheme may be applied to curved wires using a technique similar to that of [1], where the quadrature scheme is developed on a linear segment tangent to the curved segment. Here, however, the tangent linear segment is used to determine the sample points and quadrature weights to be used in the potential integrals. The linear segment is tangent to the source segment at the projection of the observation point onto the source segment axis as shown in Fig. 1. Once the weights and sample points are determined, the same quadrature algorithms used to calculate the nonsingular forms of (1)-(3) may be used with the new weights and sample points.

To see that this may be done, consider a generalized form of the integrals in (1) and (2) over each segment given by

$$
\int_{\Delta L} B\left(\mathbf{r}^{\prime}\right) K\left(\mathbf{r}, \mathbf{r}^{\prime}\right) d \sigma^{\prime}=\mathcal{J}_{0} \int_{0}^{1} B(\xi) K\left(\mathbf{r}, \mathbf{r}^{\prime}\right) \frac{\mathcal{J}}{\mathcal{J}_{0}} d \xi,
$$

where $B$ represents a basis function (or its divergence) defined on the axis of a wire segment $\Delta L$ parameterized by a normalized coordinate $\xi$ with Jacobian $\mathcal{J}=d \sigma^{\prime} / d \xi=\left|d \mathbf{r}^{\prime} / d \xi\right|$, and $\mathcal{J}_{0}$ is the Jacobian evaluated at $\mathbf{r}_{0}$, the projection of the observation point onto the curved source segment axis. A tangent vector at this point is given by [5]

$$
\ell\left(\mathbf{r}_{0}\right)=\left.\frac{d \mathbf{r}}{d \xi}\right|_{\mathbf{r}=\mathbf{r}_{0}}
$$

such that $\mathcal{J}_{0}=\left|\ell\left(\mathbf{r}_{0}\right)\right|=\ell\left(\mathbf{r}_{0}\right)$. The right-hand side of (4) now may be merely interpreted as an integration over a linear segment of length $\mathcal{J}_{0}$ with a modified integrand. This integral form is similar to that used in [4] for singularity analysis on linear wire segments. To see this, consider a Taylor series expansion of $\mathbf{r}^{\prime}$ about $\mathbf{r}_{0}$,

$$
\mathbf{r}^{\prime}=\mathbf{r}_{0}+\left(\xi-\xi_{0}\right) \ell\left(\mathbf{r}_{0}\right)+\mathcal{O}\left(\left(\xi-\xi_{0}\right)^{2}\right) .
$$

The tangent segment at $\mathbf{r}_{0}$ is merely the first two terms on the RHS of (6). Hence, the ratio of Jacobians is

$$
\frac{\mathcal{J}}{\mathcal{J}_{0}}=1+\left.\frac{\hat{\ell}\left(\mathbf{r}_{0}\right)}{\left|\ell\left(\mathbf{r}_{0}\right)\right|} \cdot \frac{d^{2} \mathbf{r}^{\prime}}{d \xi^{2}}\right|_{\mathbf{r}^{\prime}=\mathbf{r}_{0}}\left(\xi-\xi_{0}\right)+\mathcal{O}\left[\left(\xi-\xi_{0}\right)^{2}\right] .
$$

As the source point approaches the projected observation point, (6) and (7) become $\mathbf{r}^{\prime} \rightarrow \mathbf{r}_{0}$ and $\mathcal{J} / \mathcal{J}_{0} \rightarrow 1$, respectively. Hence, the singularity in the integrand in (4) is unchanged from that of a linear wire segment. In summary, since the singular behavior of the integrand on the curved wire segment is similar 
to that on a linear segment, the approach of [4] may be used to calculate the integration points and weights for use in (1) and (2).

\section{Results}

A helical antenna is constructed with a wire of radius $0.5 \mathrm{~mm}$ [6]. The helix is circular with a $25 \mathrm{~mm}$ circumference and a pitch angle of $4^{\circ}$. The antenna is attached to an infinite ground plane using two straight wire elements, each with the same radius as the helix wires. The first straight wire (length $1.25 \mathrm{~mm}$ ) is attached to the ground plane such that it is perpendicular to the ground plane. The free end of this wire is fastened to the second straight wire, which is $3.9789 \mathrm{~mm}$ and is horizontal to the ground plane. The helix is joined to this wire, forming the complete antenna. A unit delta-gap voltage source excites the structure at the base at $12 \mathrm{GHz}$. The currents shown in Figs. 2 and 3 are for cases involving 100 linear elements and 20 quadratic $(q=2)$ elements, respectively, to approximate the antenna (including the antenna image). Data from [6] are also shown in the figures. Good results are achieved with the $p=1$ (quadratic) basis functions (37 unknowns) compared to 99 unknowns with 100 straight elements.

\section{Summary and Conclusions}

A method of moments formulation using higher-order wire segments and basis functions for calculating the currents on arbitrarily-shaped wire structures is presented. This approach leverages recent novel advances in techniques for evaluating the integrable singularity. Results indicate that fewer higher-order bases are needed than the usual ten unknowns per wavelength rule of thumb. This approach is expected to be useful in advancing mesh ( $h$-adaptive) and basis function ( $p$-adaptive) algorithms for arbitrarily-shaped wire structures.

\section{References}

[1] N. J. Champagne, J. T. Williams, and D. R. Wilton, "The use of curved segments for numerically modeling thin wire antennas and scatterers," IEEE Trans. Antennas Propagation, vol. 40, no. 6, pp. 682-689, 1992.

[2] M. A. Khayat and D. R. Wilton, "Evaluation of singular and near-singular potentials," 2003 IEEE AP-S International Symposium and North American URSI Radio Science Meeting, Columbus, OH, June 2003.

[3] M. Khayat and D. R. Wilton, "Revisiting the evaluation of potential integrals," 2003 International Conference on Electromagnetics in Advanced Applications, Torino, Italy, pp. 83-86, Sept. 2003.

[4] D. R. Wilton and N. J. Champagne, "Evaluation and integration of thin wire kernels," IEEE Trans. Antennas Propagation, accepted.

[5] R. D. Graglia, D. R. Wilton and A. F. Peterson, "Higher order interpolatory vector bases for computational electromagnetics," IEEE Trans. Antennas and Propagation, vol. 45, no. 3, pp. 329-342, Mar. 1997. 
[6] H. Nakano, H. Takeda, T. Honna, H. Mimaki, and J. Yamauchi, "Extremely lowprofile helix radiating a circularly polarized wave," IEEE Trans. Antennas and Propagation, vol. 39, no. 6, pp. 754-757, June 1991.

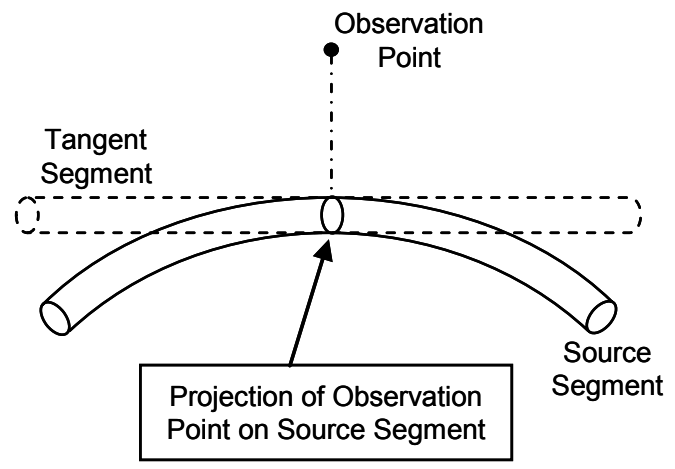

Fig. 1. Element tangent to a curved wire segment.

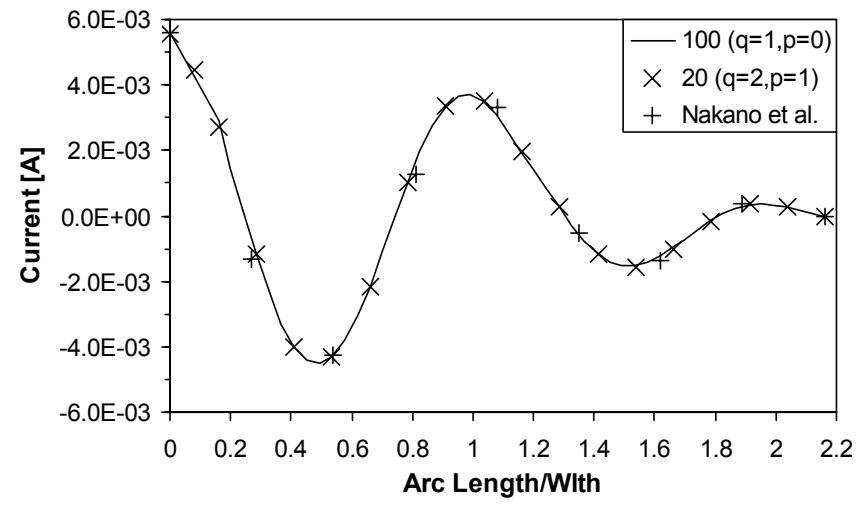

Fig. 2. Real part of the current on a helical antenna mounted on a ground plane at $12 \mathrm{GHz}$.

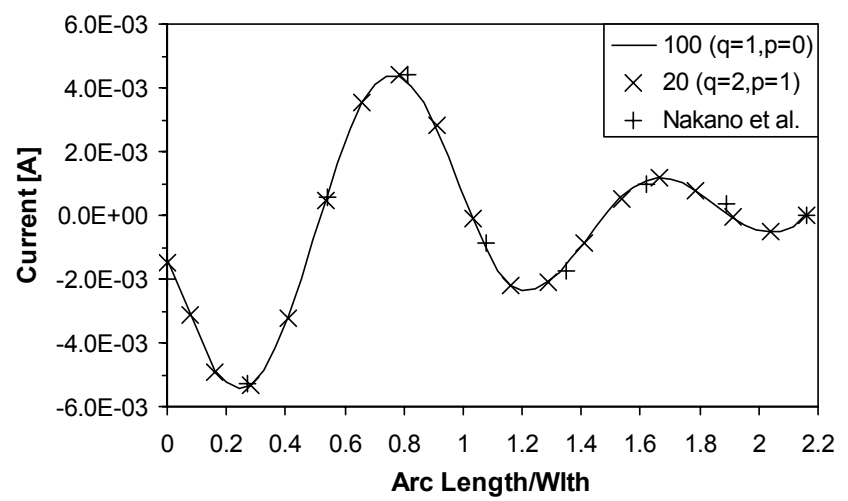

Fig. 3. Imaginary part of the current on a helical antenna mounted on a ground plane at $12 \mathrm{GHz}$. 